\title{
CASE REPORT - AN UNUSUAL PRESENTATION OF BRANCHIAL CYST WITH MEDIASTINAL EXTENSION.
}

Sanjeev Suman ${ }^{1}$, Babita $^{2}$

\section{HOW TO CITE THIS ARTICLE:}

Sanjeev Suman, Babita. "Case report - an unusual presentation of branchial cyst with mediastinal extension". Journal of Evolution of Medical and Dental Sciences 2013; Vol2, Issue 35, September 2; Page: 6651-6655.

ABSTRACT: Branchial cysts are congenital anomalies. Very few cases have been reported about branchial cysts with mediastinal extension. We report here a case of branchial cysts with mediastinal extension.

INTRODUCTION: Branchial cysts (also known as lateral cervical cysts), predominantly present in the lateral aspect of the neck. Branchial cleft cysts are congenital anomalies. Typically, a fluctuant swelling is felt deep to the sternocleidomastoid at the junction of its upper third and lower two thirds. They often present in the second and third decades of life. No racial or sexual predilection has been identified. Males and females are equally affected and there is occasionally a hereditary tendency. Diagnosis is usually made clinically. Branchial cleft cysts are benign; however, super infection, mass effect, and surgical complications account for morbidity. Patients relate its discovery to an attack of pharyngitis, ear infection, or dental infection, and many report temporary enlargement with or without tenderness during periods of upper respiratory tract infection. Inflamed cysts may progress to abscess formation with the possibility that rupture or incision and drainage will lead to either permanent sinus formation or to recurrent cyst formation and infection. Recurrence rates are reported.

CASE REPORT: A previously well 12 year old girl presented to outpatient department with a two month history of left sided neck swelling \& associated symptoms of mild discomfort in the region of neck swelling \& mild degree of odynophagia. There was no history of and respiratory compromise. Past medical history was unremarkable \& she was on no regular medications. On examination, a large about $8 \times 3 \mathrm{~cm}$ size left neck swelling noted limited posteriorly by the ipsilateral sternocleidomastoid muscle. The mass was soft in consistency, fluctuant \& non-tender. No visualized engorged veins noted over the swelling. There was no evidence of fistula. No other mass or abdominal examinations were normal with no evidence of any organomegaly. The full blood counts were within normal limits. Chest X-ray was also unremarkable. A hematological referral was made \& ultrasound of the neck was advised. USG -neck revealed a cystic mass in the left side of the neck extending inferiorly up to the ipsilateral supraclavicular region. The inferior most aspect of the lesion could not be delineated. For further evaluation a MRI scan of the neck was done on a Siemens MR scanner using IV contrast. Routine T1 wt. \& T2 wt. as well as post contrast T1 wt. images were taken in axial, sagittal \& coronal planes. Post contrast T1 wt. coronal image demonstrates a left sided neck mass with a hypointense centre \& isointense peripheral margins \& no evidence of any enhancements (Fig-1). T2 with axial image shows a hyperintense looking mass in the left side of neck limited posteriorly by the ipsilateral sternocleidomastoid muscle \& it is also extending into the left Para pharyngeal space displacing the carotid vessels (Fig-2). T2 wt. coronal image demonstrates 


\section{CASE REPORT}

the hyperintense looking left neck mass causing mild effacement of the adjacent oropharynx (Fig-3). Another T2 wt. coronal image shows evidence of superior mediastinal extension of the left sided cystic mass (Fig-4). On the basis of MR findings a possible diagnosis of left sided branchial cyst with mediastinal extension was made. The diagnosis was further supported by the histopathological correlation.

Figure - 1:

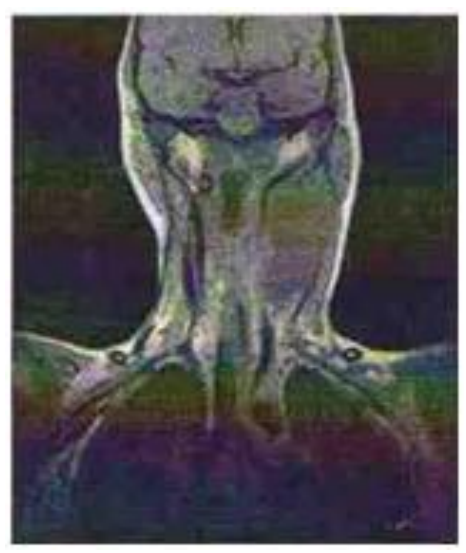

\section{Post Contrast $\mathbf{T}_{1}$ wt. coronal image showing a hypointense mass in left of neck with no evidence of enhancement.}

Figure - 2:

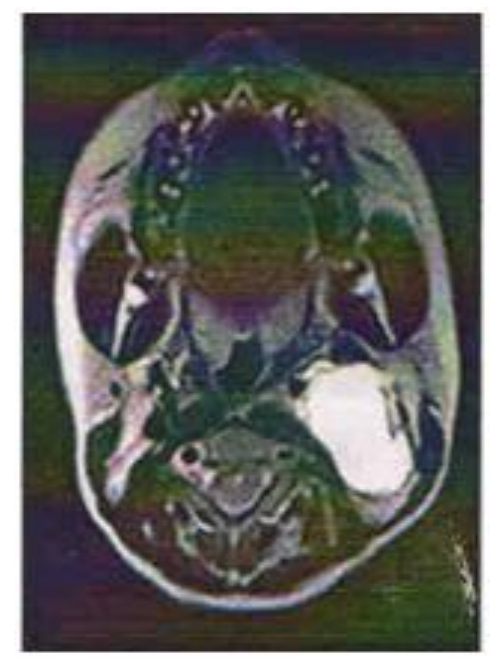

$T_{2}$ wt. axial image showing a hyper intense mass in left of neck with extension into ipsilateral parapharyngeal space \& displacement of carotid. 


\section{CASE REPORT}

\section{Figure - 3:}

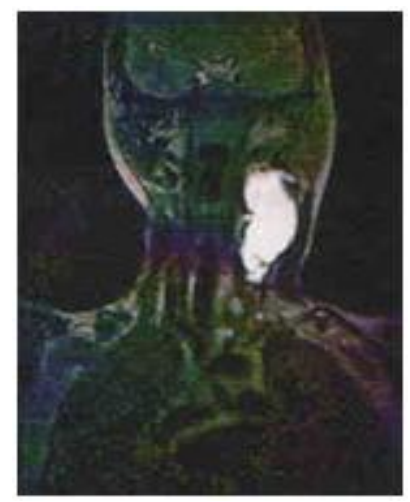

\section{$T_{2}$ wt. Coronal image showing a hyperintense mass in the left of neck with evidence of mild effacement of oropharynx.}

Figure - 4:

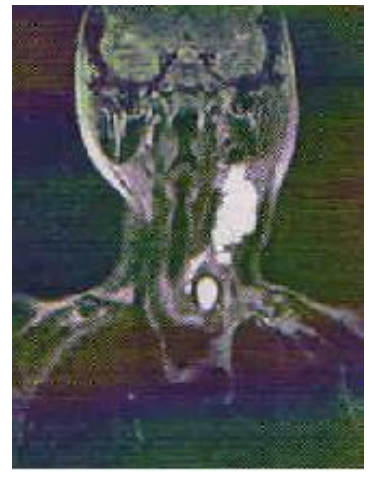

$T_{2}$ wt. coronal image of left sided branchial cyst with evidence of extension into the superior mediastinum.

DISCUSSION: The anomalies of branchial apparatus (24\%) are the second most common benign congenital masses of the neck in children after thyroglossal duct cysts (72\%). The pathophysiology of branchial cleft cysts is controversial, current theories include (a) an origin as vestigial remnants secondary to incomplete obliteration of branchial apparatus \& (b) structures that arise from "buried" epithelial cell rests. In the vestigial remnant theory, incomplete obliteration of any portion of a branchial cleft, pouch, or the cervical sinus of His can lead to formation of a sinus, fistula, or cyst. In the cell rest theory, trapped cells located anywhere in the branchial apparatus are thought to be capable of forming branchial cyst later in life 1-2. The branchial apparatus develops during the second to sixth weeks of fetal life. At the stage, the neck in shaped like a hollow tube with circumferential ridges, which are termed branchial arches. Branchial arches develop into the musculoskeletal and vascular components of the head and neck. The thinner regions between the arches are termed clefts (on the outside of the fetus) and pouches (on the inside of the fetus) ${ }^{2}$. Branchial pouches develop into the middle ear, tonsils, thymus, and parathyroid glands.

The first branchial cleft is the only cleft to give rise to and adult structure, the epithelium of the external auditory canal ${ }^{3}$. The second, third, and fourth branchial clefts merge to form the sinus 


\section{CASE REPORT}

of His, which will normally become involuted. When a branchial cleft is not properly involuted, a branchial cleft cyst forms. Occasionally, both the branchial pouch and branchial cleft fail to become involuted, and a complete fistula forms between the pharynx and skin.

FIRST BRANCHIAL CLEFT CYSTS: First branchial apparatus anomalies account for $5 \%$ to $8 \%$ of all branchial anomalies are usually in the form of a cyst or sinus ${ }^{4}$. First branchial cleft cysts are divided into type I and type II. Type I cysts are located near the external auditory canal. Most commonly, they are inferior and posterior to the tragus (base of the ear), but they may also be in the parotid gland or at the angle of the mandible. Type I cysts may be difficult to distinguish from a solid parotid mass on clinical examination. Type II cysts are associated with the submandibular gland or found in the anterior triangle of the neck. Although this anomaly occurs in childhood, it is more commonly encountered in middle aged women who present with recurrent abscesses or inflammatory processes at the angle of the mandible for ear or as recurrent parotid abscess.

SECOND BRANCHIAL CLEFT CYSTST: The second branchial cleft accounts for 95\% of branchial anomalies. Most frequently, second branchial cleft cysts are identified along the anterior border of the upper third of the sternocleidomastoid muscle and adjacent of the muscle. However, these cysts may present anywhere along the course of a second branchial fistula, which proceeds from the skin of the lateral neck, between the internal and external carotid arteries, and into the palatine tonsil. Therefore, a second branchial cleft cyst in part of the differential diagnosis of a parapharyngeal mass. Because these cysts usually present as a neck mass in the young child, sonography is often the initial imaging study. The cysts range in size from 1 to $10 \mathrm{~cm} . \mathbf{5 , 6}$

THIRD BRANCHIAL CLEFT CYSTS: Third branchial cleft cysts are rare. In spite of the rarity of third branchial cysts, they are the second most common congenital lesion of the posterior cervical space after lymphatic malformation 7. A third branchial fistula extends from the same skin location as a second branchial fistula cleft; however, a third branchial fistula coursed posterior to the carotid arteries and pierces the thyrohyoid membrane to enter the larynx, terminating on the lateral aspect of the pyriform sinus. Third branchial cleft cysts occur anywhere along that course (e.g., inside the larynx), but they are characteristically located deep to the sternocleidomastoid muscle.

FOURTH BRANCIAL CLEFT CYSTS: Fourth branchial cleft cysts are extremely rare. A fourth branchial fistula arises from the lateral neck and parallels the course of the recurrent laryngeal nerve (around the aorta on the left and around the subclavian artery on the right), terminating in the apex of the pyriform sinus; therefore, fourth branchial cleft cysts arise in various locations, including the mediastinum.

CONCLUSION: Both Computed Tomography (CT) scanning and Magnetic Resonance Imaging (MRI) are useful in the evaluation of branchial cleft cysts and for parapharyngeal masses that may be second branchial cleft cysts. The relationship of glandular tissue to the mass (e.g., fat planes between the parotid gland and a parapharyngeal mass) is important for differential diagnosis and surgical planning. Both CT scanning and MRI may be unable to distinguish a branchial cleft cyst from a lymphangioma in children. In adults, necrotic metastatic squamous cell carcinoma to cervical nodes 
\& abscesses may mimic branchial cleft cysts. Contrast study is helpful in such cases. Contrastenhanced CT scan reveals a well-defined, nonenhancing mass of fluid attenuation in a characteristic location. Necrotic lymph nodes \& abscesses usually show marginal enhancements. The location depends on which branchial cleft is cysts, but differential considerations include lymphangioma (cystic hygroma, lymphatic malformations), glandular cysts, lymph nodes, ranulas, dermoid cysts, laryngiomas (venous malformations), and pareagangliomas. Cystic metastases (e.g., papillary thyroid carcinoma), necrotic metastases (e.g., squamous cell carcinoma), and tuberculus lymphadenitis can result in low-attenuating lymph nodes. Branchial cleft cysts have high signal intensity on $\mathrm{T}_{2}$ - weighted MR-images. On $\mathrm{T}_{1}$ - weighted images, the signal intensity is usually low, but prior infection can provoke proteinaceous debris that increases the $\mathrm{T}_{1}$ signal intensity. Uninfected branchial cleft cysts should not enhance on MRI. Fluoroscopic fistulography or CT fistulography may also be used to delineate the course of branchial cleft sinus or fistula. This can aid insurgical planning and in predicting potential complications from surgery. Almost all branchial abnormalities should be excised early in life since repeated infection is common, making resection more difficult. Infected sinuses \& cysts require initial incision \& drainage.

\section{REFERENCE:}

1. Benson MT, Dalen K, Mancuso AA, et al: Congenital anomalies of the branchial apparatus: Embryology \& pathologic anatomy. Radiographic 1992; 12:942-960

2. Maran ADG, Buchanan DR: Branchial cysts, sinuses, \& fistulae, Clin Otolaryngol.1978; 3:7792.

3. Moore DM: Synovial sarcoma of the head \& neck. Arch Otolaryngol Head Neck Surg.1987; 113:311-313.

4. Harnsberger H, Mancuso A, et al: Branchial cleft anomalies \& their mimics: Computed tomographic evaluation Radiology'1994; 152:739-748.

5. Deane S, Telander R: Surgery for thyroglossal duct \& branchial cleft anomalies. Am I surg.1978;136:348-353,

6. Telander R, Filstom H: Review of head \& neck lesion in the infancy \& childhood. Surg Clin North Am.1992; 72:1429-1447.

7. Parker G, Harnsberger H, Smoker W: The anterior \& posterior cervical spaces. Semin US CT MR.1991; 12:257-273.

\section{AUTHORS:}

1. Sanjeev Suman

2. Babita

\section{PARTICULARS OF CONTRIBUTORS:}

1. Senior Resident, Department of Radiology, Patna Medical College \& Hospital, Patna.

2. Tutor, Department of Microbiology, Patna Medical College \& Hospital, Patna.

\author{
NAME ADRRESS EMAIL ID OF THE \\ CORRESPONDING AUTHOR: \\ Dr. Sanjeev Suman, \\ 0/63, Nalanda Scan Centre, \\ Doctor's Colony, Kankarbagh, \\ Patna - 800020, Bihar. \\ Email - drbabitasmn@gmail.com \\ Date of Submission: 06/08/2013. \\ Date of Peer Review: 10/08/2013. \\ Date of Acceptance: 22/08/2013. \\ Date of Publishing: 27/08/2013
}

